\title{
Design of cost-efficient Glucose meter
}

\author{
Sanjana Rao ${ }^{1}$, Sattwik Mohanty ${ }^{2}$, Chirag Amarnani ${ }^{3}$, Kausar Fakir ${ }^{4}$, Sandip Dhobale ${ }^{5^{*}}$, Trupti Agarkar $^{6^{*}}$ \\ Ramrao Adik Institute of Technology, Nerul, Navi Mumbai \\ sanjana.raviprasad99@gmail.com¹,mohanty.sattwik123@gmail.com², \\ chiragamarnani@gmail.com ${ }^{3}, \underline{\text { kausar.fakir@rait.ac.in }}^{4}$, dhobale.sandip@gmail.com ${ }^{5 *}$, \\ trupti.agarkar@rait.ac.in ${ }^{6^{*}}$ \\ *Corresponding author
}

\begin{abstract}
A blood glucose metre is a small, portable device that measures blood glucose levels. To avoid long-term complications from diabetes, careful blood glucose control is required. New blood glucose metres have a small size, a large memory capacity, blood glucose manipulation techniques, and computer-based data processing capabilities.
\end{abstract}

The Glucose Detection System is implemented in tandem with an improved Op-based Potentiostat and an automatic test system. This project's goal is to investigate an improved OP-based ThreeElectrode Potentiostat that can be used in an Electrochemical Glucose Biosensor device to achieve comparable measurement accuracy. This Project enables us to make the Glucometer CostEfficient, Affordable and Easy to Use. They hold a lot of promise in the field of bio-detection on the go systems for use in health care and biomedicine.

Keywords: Potentiostat, Glucometer, affordable, easy to use, health- care.

\section{INTRODUCTION}

A glucose metre is also available as a "glucometer," is a medical device used to determine the estimated glucose concentration in the blood. It could also be a piece of sensing elements dipping in a substance and its measurement against a glucose map. It is an important part of home blood glucose monitoring (HBGM) for people suffering from diabetes or hypoglycemia. A single drop of blood is deposited on a disposable test strip by pricking the skin with a lancet, which the metre reads and measures blood glucose levels.

Clark and Lyons proposed the enzymatic electrode of glucose oxidase in Cincinnati, Ohio, in 1962. Guilbault and Lubrano employed the amperometric method in the electrochemical in 1973 to feel the amount of hydrogen peroxide $(\mathrm{H} 2 \mathrm{O} 2)$ in order to determine the glucose concentration. The use of a metal surface reactionbased electrode without an enzyme membrane to determine glucose concentration is defined. A new material is needed. The different glucose monitors are compared and contrasted. The amperometric technology is the most widely used, however, it is more difficult to design circuits for and is not appropriate for long-term measurements.
It's impossible to construct an Amperometric with a high impedance amplifier. The transducer calculated the flow of current between a biochemically sensitive and a reference electrode, which is proportional to the concentration of electro-active analytic in the solution. The glucose can be detected using a twoelectrode (RE and WE) potentiostat due to concentration polarization and the higher degree of voltage at the RE terminal. The offset voltage was provided by the induced current flow through the RE electrode, which increased the measurement error. The CE electrode is used in the Potentiostat, a threeelectrode bio-sensor that has fast sensing, high performance, and high reliability. The CE electrode creates a current loop, while the RE electrode creates a voltage direction, reducing the over-voltage effect in $\mathrm{RE}$ and reducing measuring error.

The oxygen electrode-based $\left(\mathrm{O}_{2}\right.$-based) sensor that generates the oxidation current and the hydrogen peroxide electrode-based sensor $\left(\mathrm{H}_{2} \mathrm{O}_{2}\right.$-based) sensor, which induces the reduction current, are the most commonly electrochemical glucose biosensors with electron propagation mediators were used. The Potentiostat is a device that amplifies and reads current in a bio-signal detection system. Aerometric electrochemical sensor with three electrodes, a current metre, and a control amplifier make up the basic setup. A current proportional to the concentration of substrate is detected as an electrochemical reaction on the electrode.

The Potentiostat are a big category's electronic interface of Amperometric sensors with the ability to track many biologically analytics that are critical for the environment. In system-on-chip designs, potentiostat and interface circuits are integrated in CMOS chips to improve functionality while reducing device size.

The primary goals of a glucometer are to avoid excessive insulin secretion, excessive food intake, diabetic patient analysis and prescribed treatment, and to determine the average blood glucose value. Furthermore, the glucometers currently on the market are quite expensive and out of reach for many people. As a result, the cost-effective Glucometer is both affordable and accurate. [1-8] 


\section{SYSTEM DESIGN}

An enzymatic reaction and a detector are the two most important components of glucose meters. The enzyme part of a glucose meter is usually packed in a single-use strip or reaction cuvette in a dehydrated state. The glucose in the the patient's blood sample is rehydrated and interacts because of the enzymes to create a detectable substance. A few metres produce hydrogen peroxide or an intermediary that is capable of reacting with a dye, causing a colour shift proportional to glucose solution concentration. Other metres include enzymes in a biosensor, which generates an electron, which the metre detects. Current glucose meters use three different enzymatic reactions: glucose oxidase, glucose dehydrogenase, and hexokinase. Each enzyme has its own set of benefits and drawbacks.

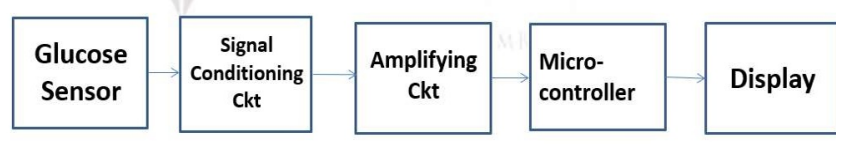

The System Design includes two parts, namely Sensor Design and Software Design.

\subsection{Sensor Design and Glucose detection:}

The below figure shows the hardware representation of the Glucometer. The Sensing Film is used to sense the amount of glucose present in the blood. The Sensing Film consists of components such as the Glucose Oxidase enzyme(GOx) immobilized on conducting polymer (CP) film deposited on intedigitated PCS board which catalyses the oxidation of glucose to hydrogen peroxide nd D-glucono-delta-lactone,

\section{Current}

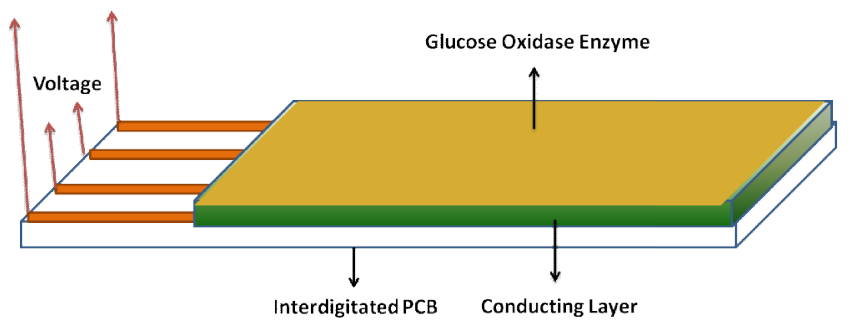

Glucose detection has been done with different glucose concentration from normal to higher levels $(100 \mathrm{mg} / \mathrm{dl}$ to $270 \mathrm{mg} / \mathrm{dl}$ ). Different glucose concentrations has been prepared in standard phosphate buffer solutions and I-V responses are recorded for different glucose concentration mentioned above.

The reactions taking place are as shown:

$$
\begin{aligned}
& \text { • Surface Reaction } \\
& 1 / 2 \mathrm{O}_{2} \rightarrow \mathrm{O} \text { (adsorbed) } \\
& \mathrm{O} \text { (adsorbed) }+2 \mathrm{e}-\rightarrow \mathrm{O}^{2-} \text { (adsorbed). }
\end{aligned}
$$

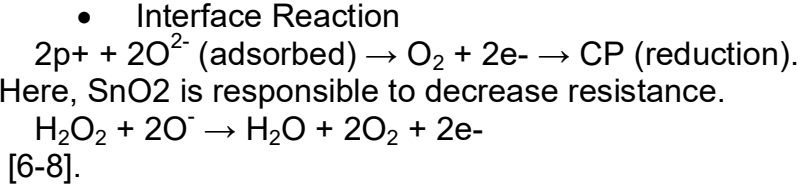

\subsection{Software Design}

The overall representation of how it would look like on the breadboard after connecting it to the Arduino UNO is as shown below: -

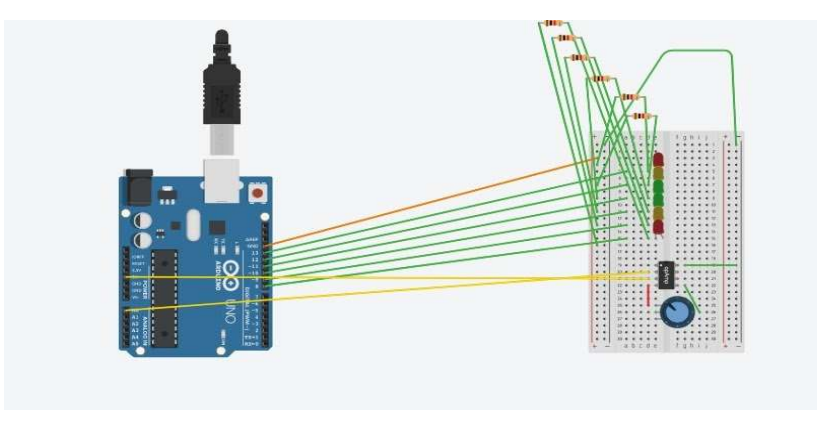

\section{GLUCOSE LEVEL STAGES}

The test for checking blood glucose can be done when one is:

- Fasting

- After Eating

- 2-3 Hrs After Eating

Glucose level according to voltage is as shown below:

\begin{tabular}{|l|l|}
\hline Voltage & Glucose Level \\
\hline Under $2 \mathrm{mV}$ & Very Low \\
\hline Between $2.8 \mathrm{mV}$ to $6.5 \mathrm{mV}$ & Low \\
\hline Between $7 \mathrm{mV}$ to $11 \mathrm{mV}$ & Normal \\
\hline Between $12 \mathrm{mV}$ to $18 \mathrm{mV}$ & High \\
\hline Greater than $18 \mathrm{mV}$ & Very High \\
\hline
\end{tabular}

\section{- Enzyme Reaction}




\section{SIGNAL CONDITIIONING CIRCUIT}

A basic glucometer consists of a signal conditioning circuit. Signal conditioning is a data acquisition procedure that is carried out with the aid of a signal conditioner instrument. This device transforms one form of electrical or mechanical signal (input-signal) into a different type of signal (output-signal) (output-signal).

A signal conditioning circuit's functions are as follows: -

i. Signal Conversion- A signal conditioner's primary function is to obtain a signal and it should be converted to a higher electrical signal level. Signal conversion is frequently used in industrial applications that use a variety of sensors to perform measurements.

ii. Linearization- Certain signal conditioners can perform linearization when the signals produced by a sensor do not have a straight-line relationship with the physical measurement. This is the process of interpreting a signal generated by software, which is commonly used for thermocouple signals.

iii. Amplifying- The process of increasing the signal in preparation for processing or digitization is known as signal amplification. Signal amplification can be accomplished in two ways: by increasing the signal-to-noise ratio or increasing the resolution of the input signal.

iv. Filtering- The frequency spectrum of the signal is filtered to include only valid data and exclude noise. Filters can be made with both passive and active components, as well as a digital algorithm.

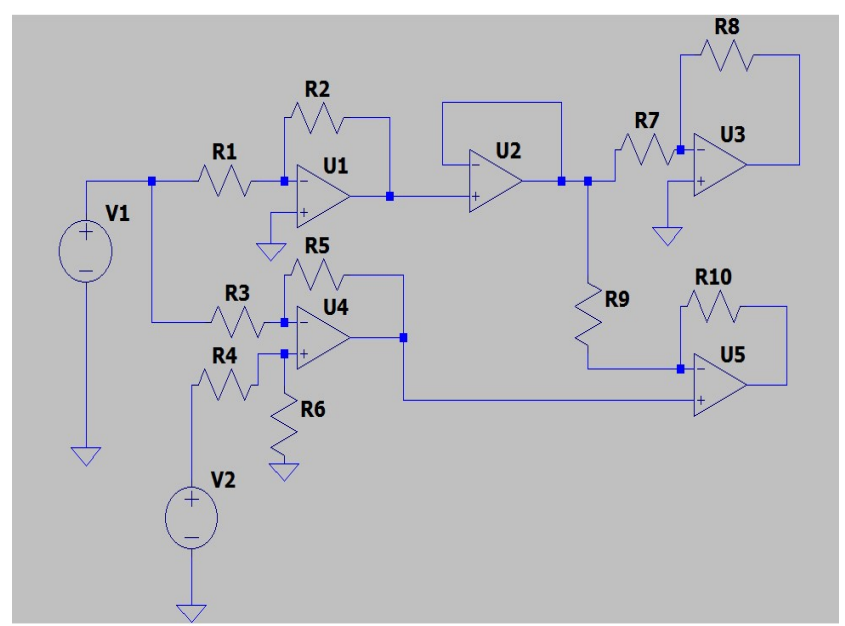

The above figure shows the signal conditioning circuit for the glucometer.

\section{WORKING OF A GLUCOMETER}

Diabetics find it difficult to keep track of their blood sugar levels, both metaphorically and literally. They prick their finger several times a day obtaining a blood droplet, which they then apply to a plastic strip that is inserted
The pancreas is usually in charge of monitoring blood sugar levels which are maintained by secreting glucagon and insulin to keep them between 100 and 200 milligrammes per deciliter of blood. However, glucose testing is a do-it-yourself proposition diabetics, either because their pancreas does not function properly or because their body is unable to process the hormones secreted by the pancreas. And it's a big one. Blood sugar levels indicate whether it is time to inject a few units of insulin - or to eat a life-saving snack.

This is where the glucometer comes into play.

Glucometers today use test strips containing glucose oxidase, an enzyme that reacts to glucose in blood droplets, as well as an interface to an electrode inside the metre. When the strip is inserted into the metre, the flux of the glucose reaction generates an electrical signal. The glucometer has been calibrated such that the number displayed in its digital display equates to the current intensity: the greater the number, the more glucose throughout the sample. Routine glucometer tests are an essential part of a patient's course of treatment, however some new systems fall far short of providing an accurate picture of real-time glucose fluctuations. [9-14]

\section{RESULTS}

The below images show the stages at which the LEDs blink after the multi-meter has been stopped at a certain voltage value. They indicate if the level of blood glucose is high, low, or regular according to the table shown in section 3 .

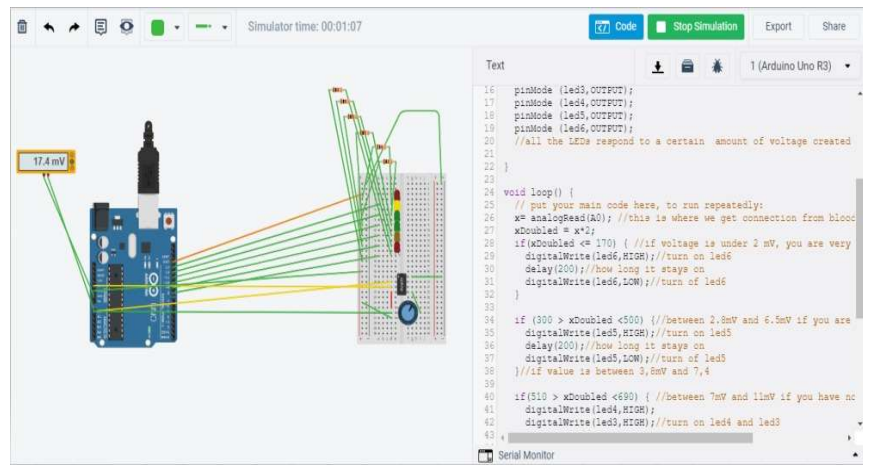

Fig: When Glucose level is high, the LED 2 (yellow) blinks

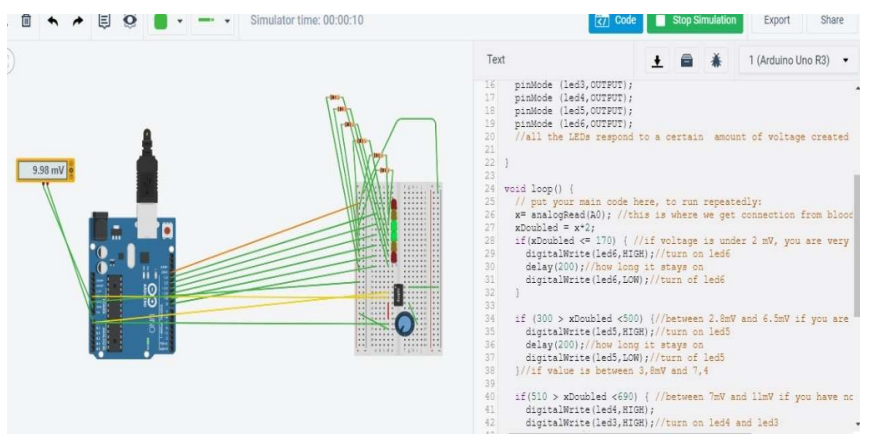




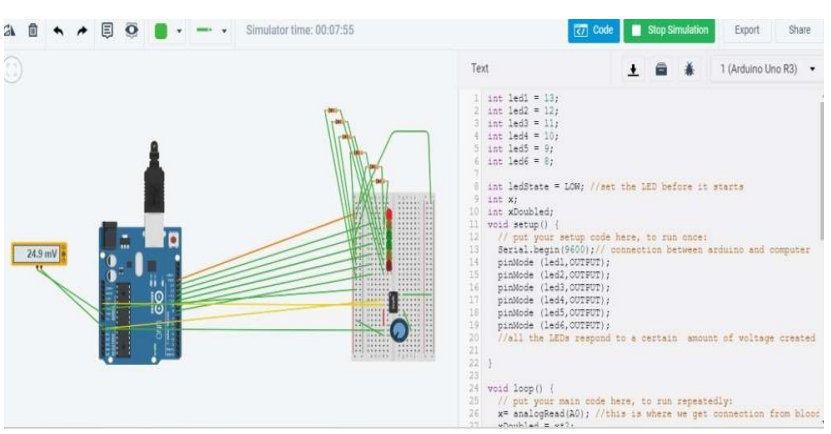

Fig: When Glucose level is very high, the LED 1 (red) blinks.

\section{ADVANTAGES}

There are many benefits of having your own blood sugar testing kit at home. These include:

The convenience of use - One of the biggest pros of a glucometer is that it offers a patient the facility of testing their blood sugar levels from the comfort of their home without needing to always go to a doctor This is especially great for patients who need to check their blood sugar levels daily. If the weather is bad - such as heavy snow - a patient need not suffer in the dark but can keep track of their blood sugar levels and collect the data or even send these across via apps to their doctor.

Possibility to make medical decisions- A glucometer gives you an accurate reading of your blood sugar level that enables you to make crucial decisions about your diet or medication. For instance, your sugar levels show as low, you might want to consume a quick snack before working out to avoid feeling sick or unconscious. Additionally, a sudden onset of high blood sugar is a warning sign that something is not right and you need medical help. This can actually make the difference between life and death.

Accuracy of reading- Based on the latest technology, modern glucometers have highly sensitive strips that are based on certain chemicals to detect every component of blood accurately. So, you can get near-to-laboratory accuracy from the comfort of your home or even while traveling.

Suitable for seniors - While everyone with diabetes should have a glucometer, the benefits are especially notable for seniors. This is because seniors often find it hard to leave their homes and head to a clinic each time that they need their blood glucose checked. Now, with glucometers, they can check their blood sugar readings at home and also share these reports with their doctors online. This ensures the good health of the patient and helps the doctors too, by easing their workload in laboratories.

Cost-effective - Finally, a glucometer is a one-time purchase. This definitely helps save money on laboratory tests for blood sugar levels. Plus, you also save money that you would spend on your commute. You do not even have to phvsicallv visit vour doctor to across online. So, overall, this is a worthwhile medical investment that is a must-have for a diabetes patient.

\section{LIMITATIONS.}

While there are many advantages, there are a few disadvantages too. They are given below: -

It hurts- Using an invasive glucometer hurts! Pain is present regardless of how small and thin the needle is. While some people become accustomed to it and thus tolerate it, it can be problematic for others. Measuring blood glucose levels is routine for diabetics, so incorporating this pain into your routine becomes difficult. This is especially true for children and adolescents with type 1 diabetes. Even mild discomfort is difficult for young kids to tolerate.

It may result in Non-Compliance - As stated earlier, using an intrusive glucometer could result in pain. Patients may develop a mental barrier as a result of the pain that inhibits them from having to check their sugar levels on a daily basis. This noncompliance can eventually affect their blood glucose control, causing hypoglycemia and hyperglycemia. Noncompliance is risky because it raises the risk of developing diabetes.

It has the potential to cause infection - The skin barrier that protects against infection is breached when you prick your finger. Diabetics are more susceptible to infection, so even the smallest gap can allow bacteria to enter and cause infection. Because diabetics have a low immune system, any infection can be dangerous. Continuous pricking at the same location can also cause skin changes. The skin at that place may become hard, making it hard to prick and acquire a blood sample.

\section{CONCLUSION}

Our main aim is to Provide a Blood Glucometer that will be determined by a variety of factors, including the patient's requirements, Easy to Utilize and Affordable.

The enhanced OP-based Potentiostat within the biodetection structure uses the glucose concentration is measured using an electrochemical enzyme-immobilized test strip. The electrode responds to the current in a proportional manner to the concentration of glucose activity. The polarity of VOX governs the role of oxidation and reduction. The difference value of $\mathrm{Rf}$ alters the measurement spectrum. It also shows Device parameters.

Cost effective glucometer is designed with signalconditioning unit and micro controller, it shows potential compatibility for glucose detection. The results obtained from the sensing kit shows good response for range glucose concentrations present in healthy person as well diabetic patient. 


\section{REFERENCES}

[1] L. Clark and C. Lyons, "Electrode Systems for Continuous Monitoring in Cardiovascular Surgery," Ann. N. Y. Acad. Sci, vol. 102, 1962.

[2] Lai, J.L., Wu, H.N., Chang, H.H. and Chen, R.J., 2011, June. Design a portable bio-sensing system for glucose measurement. In 2011 International Conference on Complex, Intelligent, and Software Intensive Systems (pp. 71-76). IEEE.

[3] Saha, Shumit and Sarker, Nayan and Hira, Avijit. (2014). Design and Implementation of a Low Cost Blood Glucose Meter with High Accuracy. 1st International Conference on Electrical Engineering and Information and Communication Technology, ICEEICT 2014. 10.1109/ICEEICT.2014.6919050.

[2] R. F. B. Turner, D. J. Harrison, and H. P. Baltes, "A CMOS Potentiostat for Amperometric Chemical Sensors," IEEE Journal of Solid-State Circuits, vol. 22, pp. 473-478, 1987.

[3] C.W. Liao. "Development of Micro Electrochemical Electrode Array Platform for Blood Sugar and Cholesterol Measurement," Master Thesis, National Taiwan University, Graduate Institute of Biomedical Engineering, Taiwan, 2005.

[4] W. Gopel and P. Heiduschka, "Interface Analysis in Biosensor design," Biosensors \& bioelectronics, Vol. 10, pp. 853-8838, 1995.

[5] J. Bard and L. R. Faulkner, Electrochemical Methods: Fundamentals and Applications, 2nd Edition, Wiley, New York, 2001.

[6] S. Dhobale, P. Joshee, G. Deore, S. L. Laware, and S. N. Kale "Nanostructured glucose-oxidase immobilized SnO2SnO2 thin films for glucose sensing", Applied Physics Letters 98, 073704 (2011)

[7] Sejin park, Hankil Boo, and taek Dong Chung, "Electrochemical Nonenzymatic Glucose sensors," Analytica Chemica Acta, Vol. 556, pp. 46- 57, 2006.

[8] Carlos Eduardo Ferrante do Amaral, Benhard Wolf, " Current Development in Non-invasive Glucose Monitoring," Medical Engineering \& Physics, Vol. 30, pp. 541-549, 2008.

[9] Audrey Sassolas, L. Blum, B.D. Leca-Bouvier, "Polymeric Luminol on Pre-treated Screen-printed Electrodes for the Design of Performant Reagentless bio-Sensor," Sensors and Actuators B: Chemical, Vol. 139, pp.214-221, 2009.

[10] Martin, S.M.,Gebara, F.H., Strong, T.D., Brown, R.B., "A Low-voltage, Chemical Sensor Interface for Systems-on-chip: the Fully-differential Potentiostat,". Proceedings of the 2004 International Symposium on Circuits and Systems, ISCAS '04, Vol.4, 23-26,

Page(s):IV - 892-5, May 2004.
[11] N. A. Latha, B. R. Murthy, U. Sunitha, " Design And Development Of A Microcontroller Based System For The Measurement Of Blood Glucose," International Journal of Engineering Research and Applications (IJERA), Vol. 2, No. 5, pp.1440-1444, Sep. 2012.

[12] M. Bularzik, D. Price, M. Rivera; "Accessible Blood Glucose Monitor," University of Connecticut, Connecticut, Biomedical Engineering Senior Design, 2006.

[13] B. Feldman, "Electrochemical Blood Glucose Test Strips for People with Diabetis," Diabetes Technol. Ther., 2003.

[14] R. Graham, "Self-Monitoring of Blood Glucose: Considerations for IntensiveDiabetes Management," A CME/CPE/CNE/CCM-Certified Supplement Tech. Rep., 2005. 